\title{
Cystic fibrosis and estrogens: a perfect storm
}

\author{
Pamela L. Zeitlin
}

Johns Hopkins University School of Medicine, Baltimore, Maryland, USA.

\begin{abstract}
Irreversible destruction and widening of the airways due to acquired infections or genetic mutations as well as those of unknown cause are more severe in females. Differences between male and female anatomy, behavior, and hormonal state have been proposed to explain the increased incidence and severity in females with airway disease such as cystic fibrosis (CF); however, a mechanism to explain a sex-related difference has remained elusive. In this issue of the JCI, Coakley et al. report that elevations in the major estrogen hormone in humans - $17 \beta$-estradiol - reduce $\mathrm{Ca}^{2+}$-activated $\mathrm{Cl}^{-}$secretion by airway epithelial cells in culture, thereby disrupting ion and water balance (see the related article, doi:10.1172/JCI33893). They measure a similar diminution of nasal epithelial $\mathrm{Ca}^{2+}$-activated $\mathrm{Cl}^{-}$secretion in women with $\mathrm{CF}$ during the menstrual cycle phase at which $17 \beta$-estradiol level is at its highest. These data suggest that for about one week of a four-week menstrual cycle, women with CF will have a reduced ability to efficiently clear airway secretions, the buildup of which is a hallmark of CF. The authors suggest that these data warrant the testing of antiestrogen therapy in females with $\mathrm{CF}$ and propose an alternative avenue for $\mathrm{CF}$ therapeutic development.
\end{abstract}

Does female sex impose genetic, hormonal, and/or behavioral constraints on lung function, including a predilection to the airway destruction and widening known as bronchiectasis? In patients with cystic fibrosis (CF), an autosomal recessive inherited disorder resulting from mutations in the CFTR that very often is associated with bronchiectasis, CFTR genotype, acquisition of airway pathogens, and environmental factors conspire to adversely affect disease outcome and survival. However, women in whom no specific genetic disease has been identified have long been recognized as being more vulnerable than men to inflammatory disorders. For example, Mycobacterium avium intracellulare (MAI) pulmonary infection (1), asthma, inflammatory bowel disease, sarcoidosis, Sjögren syndrome, and rheumatoid arthritis are predominant in women (2). Idiopathic bronchiectasis behaves differently in women than it does in men with regard to locale, incidence, coinfecting pathogens (such as MAI), and etiology. Hormonal effects have long been

Nonstandard abbreviations used: $\mathrm{CaCC}, \mathrm{Ca}^{2+}$-activated $\mathrm{Cl}^{-}$channel; $\mathrm{CF}$, cystic fibrosis; $\mathrm{ENaC}$, epithelial $\mathrm{Na}^{+}$channel; $\mathrm{FEV}_{1}$, forced expiratory volume in 1 second; NPD, nasal potential difference; UTP, uridine triphosphate.

Conflict of interest: In the previous fiscal year, the author received clinical research support from INSPIRE.

Citation for this article: J. Clin. Invest. doi:10.1172/ JCI37778. suspected to contribute to periodic (catamenial; exacerbated at the time of menstruation) pneumothoraces in women (3). In an effort to explain these observations, estrogens have been intensely studied.

Bronchiectasis is defined as a progressive abnormality of conducting bronchial airways that results in dilatation, thinning of capillary walls, and impaired mucociliary clearance (clearance of mucus by airway epithelial cell cilia). Whether the inciting event is an infection that is poorly controlled, an impairment of the immune system, or a local anatomic variant leading to inadequate mucociliary clearance, it is often recognized long after it has occurred. Treatment of infection and augmentation of mucociliary clearance can stabilize disease, but these approaches are not always effective, and certain pathogens are notorious for being difficult to eradicate. Superimpose the above triggers on a person with $\mathrm{CF}$, and you have a perfect storm.

\section{CF results from dysfunction in a cAMP-regulated $\mathrm{Cl}^{-}$channel, the CFTR}

$\mathrm{CF}$ is a systemic disorder that develops in the gastrointestinal tract prior to birth but does not manifest in the lungs until after birth. This delay in onset of airway obstruction opens a therapeutic window. CFTR is critically important to the airways and sinuses because it acts as a central regulator of periciliary ion and water content. Reduc- ing or eliminating CFTR at the apical membrane of airway epithelial cells through genetic mutation, necrotizing infections, or experimentally by siRNA disrupts cAMPmediated $\mathrm{Cl}^{-}$secretion and allows excessive epithelial $\mathrm{Na}^{+}$channel-mediated (ENaCmediated) $\mathrm{Na}^{+}$reabsorption (Figure 1 ). The net result is depleted airway surface liquid depth, poor ciliary function, impaired mucociliary clearance, and increased bacterial infections. It has long been hypothesized that parallel, non-CFTR-mediated $\mathrm{Cl}^{-}$conductance pathways (Figure 1) might serve in a redundant capacity to carry $\mathrm{Cl}^{-}$ and promote fluid secretion when CFTR is absent. A proposed member of an alternative pathway of $\mathrm{Cl}^{-}$conductance is the $\mathrm{Ca}^{2+}$-activated $\mathrm{Cl}^{-}$channel (CaCC) (4-6), which is the focus of the study reported by Coakley et al. (7) in this issue of the JCI. Unlike the outwardly rectifying $\mathrm{Cl}^{-}$channel (ORCC), which depends on CFTR to function (8), the CaCC is independent of CFTR and responsive to intracellular $\mathrm{Ca}^{2+}$ concentration. The hypothesis put forward by Coakley et al. is that the sensitivity of the $\mathrm{CaCC}$ is inversely proportional to the level of circulating $17 \beta$-estradiol, and as a result, higher $17 \beta$-estradiol levels adversely interfere with $\mathrm{CaCC}$-mediated $\mathrm{Cl}^{-}$transport across the surface of airway epithelial cells (Figure 1).

The results of previously reported experiments performed at relatively high $17 \beta$ estradiol concentrations suggest that there is precedent for suggesting that estrogens and related molecules would have an impact on airway ion transport. The most common CF-associated mutation is the deletion of phenylalanine at residue 508 in CFTR ( $\Delta$ F508 CFTR), and $17 \beta$-estradiol at $\mathrm{nM}$ concentrations has been shown to rescue $\Delta$ F508 CFTR from proteasomal degradation and increase CFTR channel activity (9). These authors identified the $17 \beta$-estradiol target as $\mathrm{Na}^{+} / \mathrm{H}^{+}$exchanger-regulator factor 1 (NHERF1). Raising $17 \beta$-estradiol levels in the medium increased the levels of NHERF1, which facilitated the trafficking of mutant CFTR to the epithelial cell surface. Another group has studied CFTR expression in a rat model of human ovarian hyperstimulation syndrome (OHSS) (10). 
A Normal airway (hydration)

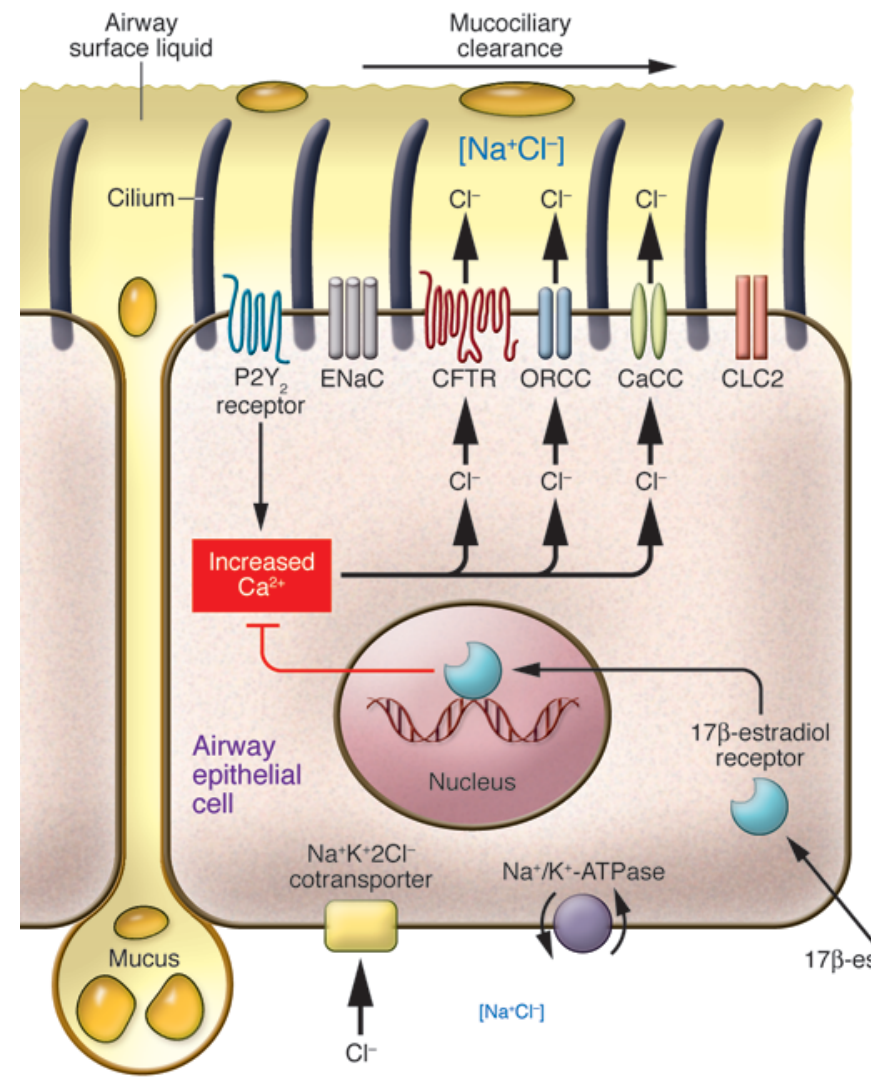

Submucosal gland
B Cystic fibrosis airway (dehydration)

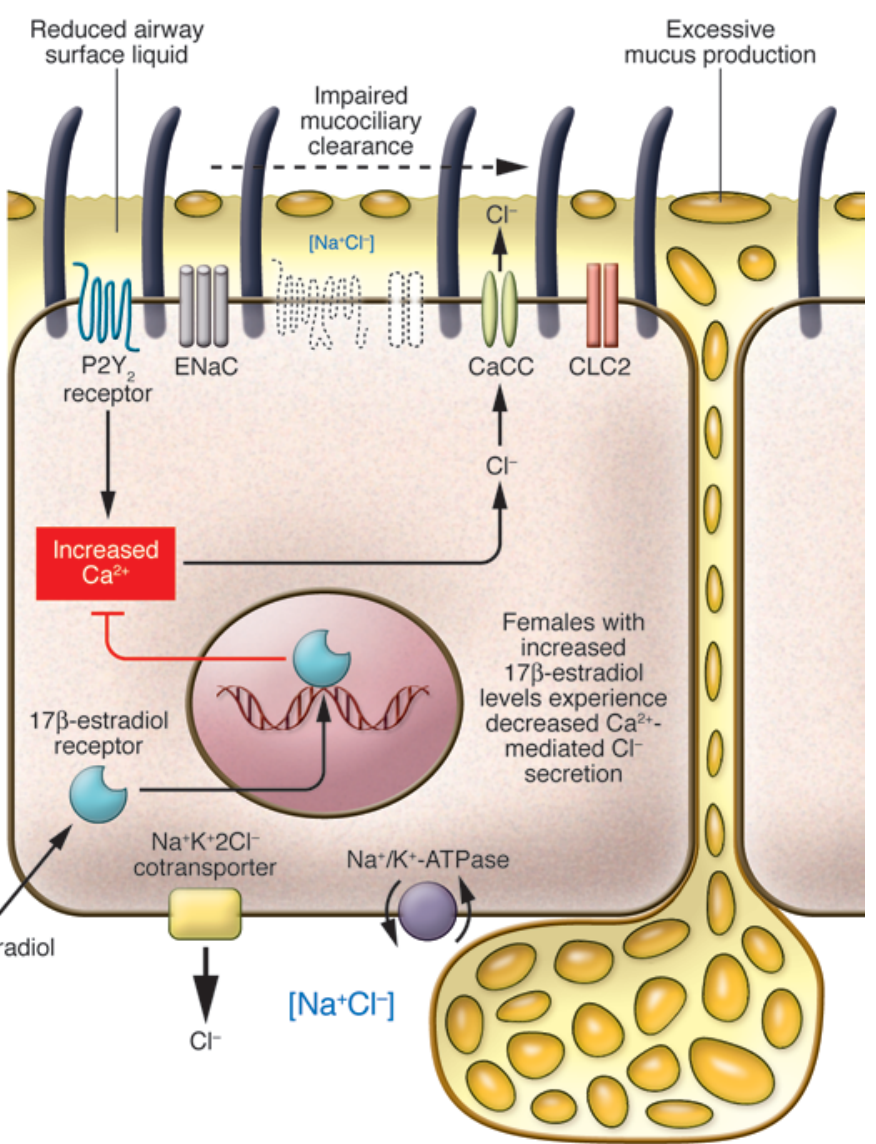

Hypertrophied submucosal gland

\section{Figure 1}

Regulation of airway surface liquid composition and depth by CFTR and ENaC in normal and CF airways. (A) In normal airways, CFTR and ENaC coexist in the apical plasma membrane of airway epithelial cells with $\mathrm{CaCC}$, outwardly rectifying $\mathrm{Cl}^{-}$channel (ORCC), and $\mathrm{Cl}^{-}$channel 2 (CLC2). Basolateral $\mathrm{Na}^{+} / \mathrm{K}^{+}-\mathrm{ATPase}$ pumps provide the driving force for active transport, and the $\mathrm{Na}^{+} \mathrm{K}^{+}+\mathrm{Cl}^{-}$cotransporter assists in moving $\mathrm{Cl}^{-}$across the basolateral membrane. Active $\mathrm{Cl}^{-}$secretion via CFTR (baseline unstimulated and agonist activated) tempers ENaC-mediated $\mathrm{Na}^{+}$reabsorption. The combination of $\mathrm{Cl}^{-}$secretion and reduced $\mathrm{Na}^{+}$reabsorption favors a healthy ion composition and depth of airway surface liquid, enabling effective ciliary beat-driven mucociliary clearance. Mucus is secreted from submucosal glands and is propelled by cilia and cough in a proximal direction. (B) In CF airways, CFTR is absent or dysfunctional and ENaC is no longer regulated, leading to hyperabsorption of $\mathrm{Na}^{+}$and an increased driving force for fluid reabsorption. The airway surface liquid depth is reduced, the submucosal glands are hypertrophied, excessive mucus is secreted, and mucociliary clearance becomes impaired. In both CF and non-CF airway epithelial cells, P2Y ${ }_{2}$ receptors coexist in the apical membrane and are stimulated by ATP or other purinergic agonists to initiate increased intracellular $\mathrm{Ca}^{2+}$. $\mathrm{Ca}^{2+}$ signaling stimulates $\mathrm{Cl}$ secretion through the $\mathrm{CaCC}$ pathway. In this issue of the $\mathrm{JCl}$, Coakley et al. (7) show that $17 \beta$-estradiol intersects with this pathway. Higher $17 \beta$-estradiol levels are sensed through estrogen receptors, and the authors show that $\mathrm{Ca}^{2+}$-activated $\mathrm{Cl}^{-}$secretion is decreased in women with $\mathrm{CF}$ at times when $17 \beta$-estradiol levels are high. The net result in CF is a worsening degree of airway surface dehydration and decreased mucociliary clearance.

OHSS occurs as a complication of assisted reproduction treatments that stimulate the ovaries. Using RT-PCR, Western blotting, and electrophysiologic techniques, this group demonstrated that CFTR expression is upregulated in this syndrome. Furthermore, estrogen but not progesterone stimulated cAMP-mediated $\mathrm{Cl}^{-}$secretion. Administration of progesterone suppressed CFTR expression and alleviated symptoms in this animal model. Exogenous $17 \beta$-estradiol but not progesterone administered to ovariec- tomized rats increases CFTR expression in uterine tissue (11). In extrapulmonary guinea pig ventricular myocytes, $17 \beta$-estradiol has been shown to potentiate $\mathrm{CFTR}^{-} \mathrm{Cl}^{-}$rents (12). The cAMP-activated $\mathrm{Cl}^{-}$current in cardiac myocytes responds to exogenous $17 \beta$-estradiol in a dose-dependent relationship at $\mu \mathrm{M}$ concentrations. However, there are opposing data regarding the effects of estrogens on CFTR-mediated $\mathrm{Cl}^{-}$secretion. Singh et al. (13) studied forskolin-activated (cAMP-activated) $\mathrm{Cl}^{-}$currents in T84 human intestinal epithelial cells. The inhibition constant $\left(K_{\mathrm{i}}\right)$ for $17 \beta$-estradiol was $8 \mu \mathrm{M}$, which is unlikely to be experienced in vivo under normal circumstances. Synthetic estrogens and the selective estrogen receptor modulator tamoxifen also inhibited $\mathrm{Cl}^{-}$currents in these cells. The balance of the data discussed here, which employed a variety of experimental systems, suggests that CFTR expression and function are stimulated by estrogens, except in the human colon carcinoma T84 cell line. 


\section{A surrogate marker of airway epithelial ion transport}

The study reported by Coakley et al. (7) in this issue of the JCI employed the in vivo nasal potential difference (NPD) assay to measure the activity of $\mathrm{Cl}^{-}$channel pathways. NPD is a surrogate biomarker of $\mathrm{Na}^{+}$and $\mathrm{Cl}^{-}$transport in the lower airways $(14,15)$. NPD measurements of $\mathrm{Na}^{+}$reabsorption and the activity of various agonist-activated $\mathrm{Cl}^{-}$secretory pathways have informed therapeutic development of gene therapy vectors (15), $\mathrm{Na}^{+}$channel inhibitors (16), alternative $\mathrm{Cl}^{-}$channel activators (17), and CFTR repair molecules (18). The test is performed in the clinic and requires the subject to be free of recent nasal steroids, upper respiratory infection, or topical adrenergic agents. Using this assay, it is possible to quantify the activity of the $\mathrm{ENaC}$ and several different $\mathrm{Cl}^{-}$conductance pathways, including CFTR.

Coakley et al. (7) recorded the NPD in non-CF and CF women during different phases of the menstrual cycle, during which estrogen levels naturally rise and fall. They modified the standardized NPD protocol, which is designed to measure CFTR through isoproterenolinduced increases in cAMP, by adding a perfusion with uridine triphosphate (UTP), an agonist of the G protein-coupled receptor $\mathrm{P}_{2} \mathrm{Y}_{2}$. UTP leads to increased intracellular $\mathrm{Ca}^{2+}$ concentration and subsequent $\mathrm{Ca}^{2+}$-activated $\mathrm{Cl}^{-}$secretion. The authors detected as much as a $50 \%$ reduction in the UTP-stimulated NPD during the phase of the menstrual cycle when $17 \beta$-estradiol levels were at their highest in $\mathrm{CF}$ and non-CF women. But they did not observe an increase in the isoproterenol-mediated $\mathrm{Cl}^{-}$transport portion of the NPD in non-CF or CF female subjects during the periods of the menstrual cycle in which $17 \beta$-estradiol levels were high. The most likely explanation for this is that endogenous $17 \beta$-estradiol levels are not high enough to stimulate CFTR expression and function. Another possibility is that the variability of the NPD limits the ability to detect modest increases in nasal epithelial CFTR expression. Yet the NPD has been sensitive enough to show significant differences in the amiloride-insensitive $\mathrm{Na}^{2+}$ potential (19) and now the UTPactivated $\mathrm{Cl}^{-}$secretory pathway. To further understand the effects of $17 \beta$-estradiol on ion conductance pathways, Coakley et al. (7) studied the effects of $17 \beta$-estradiol in human $\mathrm{CF}$ and non-CF airway epithelial cell cultures in vitro. They did not find a $17 \beta$-estradiol-mediated change in estrogen receptor activity. In addition, instead of a direct effect of $17 \beta$-estradiol on $\mathrm{Ca}^{2+}$ activated $\mathrm{Cl}^{-}$secretion, the study implicated upstream targets such as $\mathrm{Ca}^{2+}$ signaling rather than the $\mathrm{CaCC}$ itself. Estrogens have been studied in the context of ENaC activity, and Sweezey et al. (19) have observed stimulation of amiloride-insensitive $\mathrm{Na}^{+}$ potential difference, which, if experienced during the Coakley et al. study, would have been expected to lead to further polarization of the baseline NPD. In an earlier study, serum levels of progesterone and estrogen were measured during the menstrual cycles of 7 women with CF, and NPD values were simultaneously recorded. In this small study, estrogen varied between approximately 100 pM and 300 pM (well below the concentrations used in vitro in most studies) and progesterone varied from approximately 1-40 mM (19). Most studies use exogenous estrogens at very high levels, and the results under these artificial conditions may not extrapolate well to native conditions.

\section{Importance of the balance between $\mathrm{Na}^{+}$and $\mathrm{Cl}^{-}$transport in regulation of airway surface liquid homeostasis}

Which kind of ion channel is most important for periciliary ion and water content CFTR, CaCC, or ENaC? This debate continues to rage. CFTR-null mice do not develop lung disease without a superimposing infection, yet mice overexpressing $\mathrm{ENaC}$ immediately suffer from airway inflammation (20). Unfortunately, the CaCC has not been convincingly cloned, although a candidate subunit has been identified (4-6), and knockout of the CaCC cannot be studied. Clearly, autosomal recessive classic $\mathrm{CF}$ is most serious for humans, followed by $\mathrm{ENaC}$ gain-of-function disease $(\mathrm{ENaC}$ is a product of the non-voltage-gated $1 \gamma$ [SNNC1G]). Can a channel or channels regulated by nucleotides and inhibited by high $17 \beta$-estradiol levels during specific phases of the menstrual cycle have an impact on the incidence and severity of airway disease in women? Coakley et al. (7) speculate that antiestrogen therapy (such as tamoxifen) should be tested in women with CF to promote maximal non-CFTR-mediated $\mathrm{Cl}^{-}$regulation. However, we must consider whether the known side effects, such as endometrial cancer, pulmonary embolism, deep-vein thrombosis, stroke, uterine abnormalities, and cataracts, outweigh the potential benefits of this proposed therapy. The authors predict that for one week out of every four of a woman's menstrual cycle, airway mucociliary clearance will be compromised by a decrease in $\mathrm{CaCC}$ function. Is this cyclical compromise in mucociliary clearance responsible for diminished female survival in CF? A small study of 12 women with CF compared forced expiratory volume in 1 second $\left(\mathrm{FEV}_{1}\right)$ at time of ovulation (high $17 \beta$-estradiol and low progesterone levels) to that during the luteal phase (high $17 \beta$-estradiol and high progesterone levels) and during the menstrual phase (low $17 \beta$-estradiol and low progesterone levels) (21). FEV 1 was significantly higher during the luteal phase as compared with $\mathrm{FEV}_{1}$ during ovulation and menstruation. These authors interpreted their findings to suggest that lung function changes were related to progesterone levels, based on their earlier studies of delayed onset of puberty in CF girls (22).

Sex-based differences have been described for a number of aspects of CF. Some of these differences may be the result of hormonal exposures or genetic inheritance. Others may be sociological or behavior based. Over the past few decades, there has been an increased mortality reported for women with CF (23); however, this sex-related difference may be dissipating as more attention is given to aggressive therapy, as discussed in Coakley et al. (7). Of the causes of mortality, lung infections are described more often for women with CF (23). In data collected from 1995 to 2005 in Ireland (24), both male and female adults with CF were more likely to die if they had worse lung function and were infected with either Pseudomonas aeruginosa or Burkholderia cepacia complex. $\mathrm{FEV}_{1}$ and infection with P. aeruginosa or B. cepacia are the most significant predictors of survival (24). Looking at the bulk of studies over the past two decades, women with CF have a worse prognosis overall; they participate less in aerobic exercise, ingest fewer kcals, perform less physical therapy, exhibit an accelerated decline in $\mathrm{FEV}_{1}$ with acquisition of $P$. aeruginosa, and show increased asthma reactivity.

Mucociliary clearance in particular is an important defense mechanism in CF lung disease. Female anatomy may impose increased vulnerabilities toward impaired mucociliary clearance. For example, women have smaller lungs than men relative to their height, and comparison of females and males with similar lung volumes shows that female airways are smaller. The smaller 
lung volume may result from a proportionate reduction in strength, limiting expansion and ventilation. Although women have higher airflows relative to lung volume, sex-related differences in anatomy can predispose to increased particle deposition and reduced particle clearance. Preexisting structural lung disease or compromised local immunity due to excessive mucoid secretions, abnormal composition of airway surface liquid, and airway damage may lead to increased colonization and infection with pathogens frequently seen in CF, such as nontuberculous mycobacteria (1).

\section{Therapeutic implications}

Coakley et al. (7) demonstrate that $17 \beta$ estradiol affects $\mathrm{Ca}^{2+}$ signaling, not $\mathrm{CaCC}$ conductance. The $\mathrm{P}_{2} \mathrm{Y}_{2}$ receptor agonist denufosol (25) activates CaCC conductance through the same pathway as UTP. These data point to an $17 \beta$-estradiol-mediated blockade of $\mathrm{Ca}^{2+}$ signaling distal to the purinergic receptor, raising the concern that the investigative product denufosol may have less efficacy for women during the high-17 $\beta$-estradiol periovulatory period of their menstrual cycles. Antiestrogen therapy might restore the ability to respond to $\mathrm{P}_{2} \mathrm{Y}_{2}$ agonists but at the cost of potential side effects. One alternative to antiestrogen therapy might be alternative downstream releasers of $\mathrm{Ca}^{2+}$ such as Moli1901 (lancovutide) (17). Another might be activation of a third parallel alternative $\mathrm{Cl}^{-}$channel such as the $\mathrm{pH}$ - and voltage-activated $\mathrm{Cl}^{-}$channel 2 (CLC2) (Figure 1), which can be stimulated by a prostone agonist such as lubiprostone (26) or the related investigational drug cobiprostone (www.sucampo.com/inthepipeline.html). Many of these agents are not yet approved for human use and are still in clinical development. Rather than attempting to compensate for loss of the $\mathrm{CaCC}$, it might be possible to utilize $\mathrm{ENaC}$ antagonists and reduce the driving force for periciliary fluid reabsorption (27). Investigational $\mathrm{ENaC}$ antagonists in development for CF lung disease include long-acting amiloride analogs, prostasin inhibitor QAU145 (28), and INO-4995 (27).

In summary, female sex-based vulnerabilities in CF, such as pregnancy-related declines in lung function and compro- mised nutrition and accelerated declines in lung function beginning at puberty, have long been discussed. More recent studies suggest that the gap in lung function and prognosis between women and men is narrowing. If sex hormone cycling is leading to a significant reduction in airway mucociliary clearance, perhaps low-dose oral or patch contraceptives could be modified to reduce the disadvantage. The results of the current study by Coakley et al. (7) reinforce that there is clearly a pressing need to raise awareness of sex-related differences in lung disease.

\section{Acknowledgments}

The author thanks Scott Blackman for valuable comments on this manuscript. The author is supported by NIH grants R01 HL59410, N01 BAA HL 0204, and CTSA UL1 RR 025005 as well as Cystic Fibrosis Foundation grant ZEITLIY03.

Address correspondence to: Pamela Zeitlin, Johns Hopkins School of Medicine, 200 N. Wolfe Street, Room 3051, Baltimore, Maryland 21287, USA. Phone: (410) 955-2035; Fax: (410) 955-1030; E-mail: pzeitlin@jhmi.edu.

1. Sexton, P., and Harrison, A.C. 2008. Susceptibility to nontuberculous mycobacterial lung disease. Eur. Respir. J. 31:1322-1333.

2. Morrissey, B.M., and Harper, R.W. 2004. Bronchiectasis: sex and gender considerations. Clin. Chest Med. 25:361-372.

3. Parker, C.M., Nolan, R., and Lougheed, M.D. 2007. Catamenial hemoptysis and pneumothorax in a patient with cystic fibrosis. Can. Respir. J. 14:295-297.

4. Schroeder, B.C., Cheng, T., Jan, Y.N., and Jan, L.Y. 2008. Expression cloning of TMEM16A as a calcium-activated chloride channel subunit. Cell. 134:1019-1029.

5. Caputo, A., et al. 2008. TMEM16A, a membrane protein associated with calcium-dependent chloride channel activity. Science. 322:590-594.

6. Yang, Y.D., et al. 2008. TMEM16A confers receptor-activated calcium-dependent chloride conductance. Nature. doi:10.1038/nature07313.

7. Coakley, R.D., et al. 2008. 17 $\beta$-Estradiol inhibits $\mathrm{Ca}^{2+}$-dependent homeostasis of airway surface liquid volume in human cystic fibrosis airway epithelia. J. Clin. Invest. 118:4025-4035.

8. Egan, M., et al. 1992. Defective regulation of outwardly rectifying $\mathrm{Cl}^{-}$channels by protein kinase A corrected by insertion of CFTR. Nature. 358:581-584.

9. Fanelli, T., et al. 2008. Beta-oestradiol rescues DeltaF508CFTR functional expression in human cystic fibrosis airway CFBE41o- cells through the up-regulation of NHERF1. Biol.Cell. 100:399-412.

10. Ajonuma, L.C., et al. 2005. Estrogen-induced abnormally high cystic fibrosis transmembrane conductance regulator expression results in ovarian hyperstimulation syndrome. Mol. Endocrinol. 19:3038-3044.

11. Rochwerger, L., and Buchwald, M. 1993. Stimulation of the cystic fibrosis transmembrane regulator expression by estrogen in vivo. Endocrinology. 133:921-930

12. Goodstadt, L., Powell, T., and Figtree, G.A. 2006. 17beta-estradiol potentiates the cardiac cystic fibrosis transmembrane conductance regulator chloride current in guinea-pig ventricular myocytes. J. Physiol. Sci. 56:29-37.

13. Singh, A.K., et al. 2000. Estrogen inhibition of cystic fibrosis transmembrane conductance regulatormediated chloride secretion. J. Pharmacol. Exp. Ther. 295:195-204.

14. Boyle, M.P., et al. 2003. A multicenter study of the effect of solution temperature on nasal potential difference measurements. Chest. 124:482-489.

15. Knowles, M.R., Paradiso, A.M., and Boucher, R.C. 1995. In vivo nasal potential difference: techniques and protocols for assessing efficacy of gene transfer in cystic fibrosis. Hum. Gene Ther. 6:445-455.

16. Knowles, M.R., Clarke, L.L., and Boucher, R.C. 1992. Extracellular ATP and UTP induce chloride secretion in nasal epithelia of cystic fibrosis patients and normal subjects in vivo. Chest. 101:60S-63S.

17. Zeitlin, P.L., Boyle, M.P., Guggino, W.B., and Molina, L. 2004. A phase I trial of intranasal Moli1901 for cystic fibrosis. Chest. 125:143-149.

18. Zeitlin, P.L., et al. 2002. Evidence of CFTR function in cystic fibrosis after systemic administration of 4-phenylbutyrate. Mol. Ther. 6:119-126.

19. Sweezey, N.B., et al. 2007. Amiloride-insensitive nasal potential difference varies with the menstrual cycle in cystic fibrosis. Pediatr. Pulmonol. 42:519-524.

20. Mall, M., Grubb, B.R., Harkema, J.R., O’Neal, W.K., and Boucher, R.C. 2004. Increased airway epithelial $\mathrm{Na}+$ absorption produces cystic fibrosis-like lung disease in mice. Nat. Med. 10:487-493.

21. Johannesson, M., Ludviksdottir, D., and Janson, C. 2000. Lung function changes in relation to menstrual cycle in females with cystic fibrosis. Respir. Med. 94:1043-1046

22. Johannesson, M., Gottlieb, C., and Hjelte, L. 1997. Delayed puberty in girls with cystic fibrosis despite good clinical status. Pediatrics. 99:29-34.

23. Rosenfeld, M., Davis, R., FitzSimmons, S., Pepe, M., and Ramsey, B. 1997. Gender gap in cystic fibrosis mortality. Am. J. Epidemiol. 145:794-803.

24. Courtney, J.M., et al. 2007. Predictors of mortality in adults with cystic fibrosis. Pediatr. Pulmonol. 42:525-532.

25. Deterding, R., et al. 2005. Safety and tolerability of denufosol tetrasodium inhalation solution, a novel P2Y2 receptor agonist: results of a phase $1 /$ phase 2 multicenter study in mild to moderate cystic fibrosis. Pediatr. Pulmonol. 39:339-348.

26. Macdonald, K.D., et al. 2008. Lubiprostone activates non-CFTR dependent respiratory epithelial chloride secretion in cystic fibrosis mice. Am. J. Physiol. Lung Cell. Mol. Physiol. doi:10.1152/ajplung.90221.2008.

27. Thelin, W.R., and Boucher, R.C. 2007. The epithelium as a target for therapy in cystic fibrosis. Curr. Opin. Pharmacol. 7:290-295.

28. NIH. Clinical trials: QAU145. www.clinicaltrials. gov/ct2/results?term=QAU145. 